\title{
Gene expression profiling of islet-like clusters differentiated from rat pancreatic stem cells in vitro
}

\author{
Lisha $\mathrm{Li}^{1}$, Furong $\mathrm{Li}^{2}$, Shuangquan $\mathrm{Gao}^{2}$ \\ ${ }^{1}$ College of Life Sciences, Peking University, Beijing, China; ${ }^{2}$ Clinical Medical Research Center, the $2{ }^{\text {nd }}$ Clinic Medicine College \\ (Shenzhen People's Hospital), Jinan University, Shenzhen, China
}

Microarray technology is becoming one of the most productive methods used to elucidate the complex interdependence present in biological systems. This technology can be used to compare nature cells with cells differentiated from stem cells to identify molecular differences between them. To date, only a few reports have analyzed differentiated cells by microarray. Here, microarray was used to identify genes abnormally expressed in differentiated islet-like clusters compared with normal pancreas islet. Successful conversion of pancreatic stem cells (PSCs) into islet-like clusters has been reported. In this study, we investigated the properties of clusters by DTZ staining and microarray. DTZ stained clusters were selected out, then mRNA expression was investigated using microarray.

In microarray there were $5 \mathbf{0 3 1}$ gene data of which the hybridization fluoresce signals from both pancreatic islet and the differentiated cells accorded with the effective signal standard. Compared with pancreatic islet, there were 1772 genes with significantly different expression in differentiated cells, of which 797 increased and 975 decreased in expression. There were 3052 gene data of which the hybridization fluoresce signals from only one kind of samples accorded with the effective signal standard. Compared with pancreatic islet, there were 1771 genes with significantly different expression in differentiated cells, of which 678 increased and 1093 decreased in expression. In the view of gene expression mode, that $44 \%(3 \mathrm{543} / 8 \mathrm{083})$ effectively detected genes exist difference in expression reflects huge disparities in biological characteristic between 2 kinds of samples. Microarray analyses indicate that genes with obvious different expression relate to transcriptional control, regulation of cell growth, metabolism, adhesion, proliferation and so on. Loss/reduced levels of transcripts associated with pancreatic-specific lineage in differentiated cells suggest that differentiation from PSCs toward functional pancreatic islet in vitro is not efficient.

Keywords: pancreatic stem cells (PSCs), differentiate, islet-like clusters, microarray

Cell Research (2008) 18:s150. doi: 10.1038/cr.2008.240; published online 4 August 2008

Correspondence: Lisha Li

E-mail: Emily_li_happy@yahoo.com.cn 